\title{
Variation in hue of a contour-contingent aftereffect due to color adaptation during inspection of the stimulus patterns*
}

\author{
JOY HIRSCH† and GERALD M. MURCH \\ Portland State University, Portland, Oregon 97207
}

The McCollough Effect (a colored line-orientation-contingent aftereffect) has been attributed to the presence of edge detectors specific to wavelength in the human visual system. The present study tests this hypothesis by introducing unlined colored fields into the inspection condition and by comparing the subsequent aftereffect with the aftereffect induced by the inspection condition not including the unlined colored fields. The data indicate that the hues of the aftereffects differ, suggesting that the color and line stimuli may be processed by different populations of neural elements, and that the color-coded edge detector model is not adequate to account for the observations.

After viewing a pattern of vertical bars on an orange ground alternating with a pattern of horizontal bars on a blue ground, most observers report the appearance of a bluish coloration on the vertical portion of a test pattern (adjacent vertical and horizontal grid patterns on white ground) and a yellow-orange coloration on the horizontal portion. The effect is known as the McCollough Effect.

McCollough advanced an explanation known as the color-coded edge detector model to account for the occurrence of the effect. She suggested (McCollough, 1965) that the colored aftereffect may depend upon color adaptation of a population of neural elements specific to colored edges of a particular orientation. Accordingly, vertical edge detectors sensitive to orange adapt during inspection such that when vertical edges are presented on an achromatic ground, only those nonadapted color-line detectors respond, creating the perception of the approximately complementary color, blue. Similarly, horizontal edge detectors specific to blue adapt during inspection such that response to achromatic horizontal edges creates the perception of vellow-orange.

McCullough and Clark (1970) ${ }^{1}$ observed that the aftereffect of orange right diagonal was influenced by the left diagonal color that was alternated with it. The aftereffect of the orange right diagonal tended toward blue when the orange stimulus was

* This research was supported by Research Grant NSF GB-11817 from the National Science Foundation, Division of Psychobiology. The authors wish to express their appreciation to Barbara Breck for her aid in data collection.

tResearch was conducted as partial fulfiliment of the requirements for the Master of Science degree, Portland State University. Now at Columbia University Department of Psychology, New York, New York 10027 . alternated with a blue left diagonal stimulus pattern and tended toward green when the orange stimulus was alternated with a green left diagonal pattern. In keeping with the color-coded edge detector model, McCollough and Clark suggested that the perceived color was induced by wavelength adaptation of wavelength-sensitive edge detectors, which may influence the hue of the aftereffect on the orthogonal test pattern when the spectral transmissions of the two inspection patterns overlap. Therefore, the aftereffect of orange right diagonal depends upon the components of orange light not present in the alternating pattern. ${ }^{2}$

A partial test of the color-coded edge detector explanation of the McCollough and Clark observations would be made by presenting a colored field (no lines) to $\mathrm{Ss}$ before presentation of the color-line stimulus. If wavelength and edge stimuli were processed by a single population of neural elements sensitive to both, as indicated by the color-coded edge detector model, then the color aftereffect on the lined test patterns would not be affected by the presentation of an unlined color field during inspection. However, if the colored line-contingent aftereffect was created by separate systems processing the color stimuli and the line stimuli, then the "effective" color component of the lined stimulus pattern would be that portion of the spectrum not stimulated by the preceding color field. The color of the aftereffect would be approximately complementary to the nonadapted (effective) population of color receptors stimulated by the lined inspection pattern.

In the experiment Ss observed two inspection conditions. Condition 1 alternated orange vertical with blue horizontal, and the aftereffect created was measured by colorimeter. The same stimulus patterns were employed in Condition 2, where each was preceded by a plain color field that stimulated a portion of the spectrum illuminated by the following lined stimulus. The aftereffect observed was compared with the aftereffect of Condition 1.

\section{METHOD}

Stimulus and test patterns were projected onto a viewing screen via Kodak Carousel projectors located $10 \mathrm{ft}$ in front of the Ss. Each S participated individually in two inspection conditions, the second of which occured 1 week after the first. In Condition 1, Ss viewed a pattern of black vertical lines on an orange ground (Wratten Filter 16, spectral transmission: $520-700 \mathrm{~nm}$ ) alternated with a pattern of horizontal lines on a blue-green ground (Wratten Filter 47, spectral transmission: $400-520 \mathrm{~nm}$ ). In Condition 2, Ss viewed a series of four inspection stimuli: yellow field (Wratten Filter 74, spectral transmission: $510-580 \mathrm{~nm}$ ), orange vertical (same as Condition 1), blue field (Wratten Filter 47B, spectral transmission : $400-500 \mathrm{~nm}$ ), and blue-green horizontal (same as Condition 1). All stimulus patterns subtended $11.6 \mathrm{deg}$ of visual angle with an intensity of $10 \mathrm{fc}$ measured at the projection screen. The black lines of the lined patterns subtended $12.4 \mathrm{sec}$ of arc with the colored area

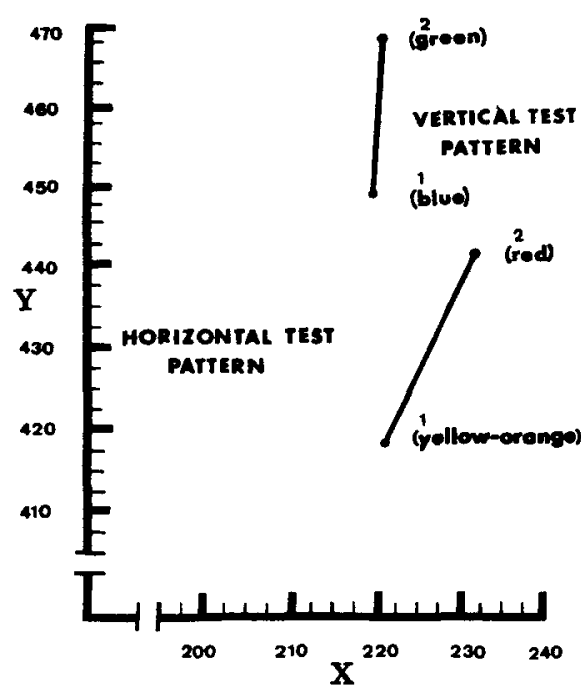

Fig. 1. Mean CIE $x, y$ coordinates for color matches of vertical and horizontal test patterns for Conditions 1 and 2. Vertical test pattern coordinates: Condition $1, .220, .449$; Condition 2, .223, .469. Horizontal test pattern coordinates: Condition 1 , $.223, .417$; Condition $2, .234, .442$. 


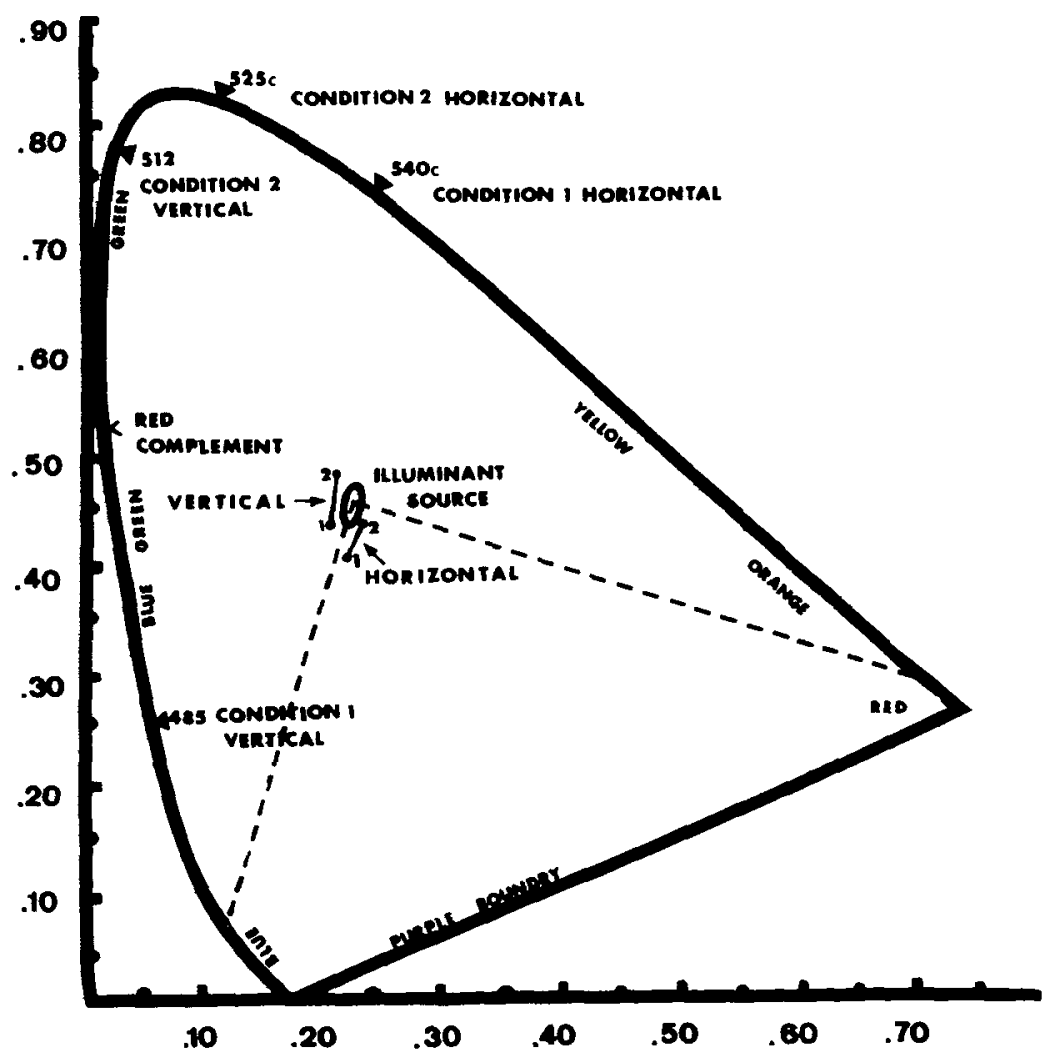

Fig. 2, Chromaticity diagram showing interpolated dominant wavelengths of mean color matches (Conditions 1 and 2) on horizontal and vertical teat patterns. The area inside the dotted line defines hues that cannot be obtained by mixing white light with a single spectrum color. Dominant wavelengths of the matches in this area are specified by their color complement.

between the lines subtending $24.9 \mathrm{deg}$ of arc.

In both conditions, each inspection pattern was shown for $10 \mathrm{gec}$ with a .1 rec interstimulus interval and was alternated 20 times. Following the inspection condition, $S$ waited in the unlighted test room for a period of time until any negative afterimage was extinguished, at which time test patterns of vertical and horizontal lines were projected onto the screen. The dimensions and intensity of these patterns were equated to the stimulus patterns. Se were asked to name any color perceived on the line patterns and to match the colored aftereffect by a tristimulus projection colorimeter (Murch, 1971). Color matches of the aftereffects were measured by three photometer readings indicating percent transmittance of red, blue, and green in each match.

In order that no residual effect persist from one experimental period to the other, $S$ s observed a "disinhibition" condition at the close of each sescion. Stimulus colors and line orientations were reversed, and a single alternation of the patterns was presented. Allowing time for the extinction of a negative afterimage, $s$ turned on the test patterns and named the color of the two patterns. The procedure was repeated until $S$ indicated that the test patterns appeared achromatic.

Ss were 28 volunteers, each screened for normal color vision, from a perception class. Matching reliability was determined for each $\mathbf{S}$ with the colorimeter prior to the initial experimental session. So viewed a total of three filters each of which was projected onto the screen four times (the order of presentation was counterbalanced) and matched each color successively. Settings were recorded for each match on the vernier acale of the colorimeter, and standard deviations on each filter per $S$ were determined. Only Ss demonstrating competence in using the colorimeter participated in the experiment.

Photometer readings were translated in CIE $x, y$ coordinates, and the means plotted on a chromaticity diagram. In order to identify colorimetric differences, four dependent variables were employed: $x$ coordinate, $y$ coordinate, resultant vector, and tangent. The $x$ and $y$ values were coordinates of each color match plotted in chromaticity space. The resultant vector was determined by vectorially adding the $x$ and $y$ components of the matches indicating distance from the origin. In order to obtain a measure of the direction of each color point, the tangent was found by dividing the $y$ coordinate by the $x$ coordinate. $T$ values were derived from individual difference scores on each dependent variable per test pattern.

\section{RESULTS}

The apparent hues observed by the So were summarized by taking the mean $x$ and $y$ coordinates for each condition per test pattern and plotting respective points. Seven Ss reported no colored aftereffect on the test patterns, which was confirmed by their matches and, consequently, were not included in the results.

Figure 1 shows mean $x$ and $y$ values for Conditions 1 and 2 per teat patterm plotted in chromaticitv sace. Perceptually, the vertical matches changed from a bluish hue in Condition 1 to a greenish hue in Condition 2, and the horizontal matches altered from a yellow-orange hue to one more reddish. This shift in hue of the aftereffect was confirmed by the interpolated dominant wavelength of the mean matches. As indicated in Fig. 2, the mean dominant wavelength of matches for Condition 1 on the horizontal text test pattern was $485 \mathrm{~nm}$ (blue) and for Condition 2, $512 \mathrm{~nm}$ (green). Dominant wavelength of mean matches for Condition 1 on the horizontal test pattern was $540 \mathrm{c} \mathrm{nm}$ and for Condition 2, $525 \mathrm{c} \mathrm{nm}$. (Due to their position on the chromaticity diagram, the dominant wavelengths for the horizontal matches are represented by their color complements).

A t-test for correlated observations (one-tailed) was used to test the statistical significance of the match differences between Conditions 1 and 2. The points plotted in Fig. 2 for the horizontal test pattern are significantly different on the $x$ coordinate $(t=-2.274, d f=20, p=.01)$, and on the resultant vector of the matches ( $t$ $=-1.810$, df $=20, p=.05$ ). Point plotted for Conditions 1 and 2 on the vertical test pattern are significantly different on the tangent $(t=1.382$, df $=20, p=.10$ ) and the $y$ coordinate $(t=-1.167, d f=20, p=.20)$.

\section{DISCUSSION}

While McCollough and Clark suggested that the aftereffect may be due to a "linkage" between left and right border detectors that produced a direct effect of the left-diagonal inspection color upon right diagonal detectors, the present study shows that their results may have been due to 
general color adaptation independent of edge detectors. The data show that the aftereffect on the vertical test pattern tended toward green or blue, depending upon the spectral properties of the preceding unlined pattern and, similarly, the aftereffect on the horizontal test pattern tended toward yellow or red, depending upon the spectral properties of the preceding color field stimulus. These same variations in aftereffect hue were observed by McCollough and Clark, using only colored line stimulus patterns, suggesting that their results may have been also produced by selectively preadapting the color vision system, not necessarily color-coded edge detectors.

The results indicate that this colored line-contingent aftereffect may be created by at least two levels in the visual system: color receptors independent of slope analyzers. Consequently, the color-coded edge detector model is not adequate to account for the occurrence of the McCollough Effect.

\section{REFERENCES}

MCCOLLOUGH, C. Color adaptation of edge detectors in the human visual system. Science, $1965,149,1115-1116$.
MURCH, G. M, CIE $x, y$ coordinates from an inexpensive projection colorimeter. 1971 , in press.

STROMEYER, C. F. Further studies of the $M$ cCollough effect. Perception \& Psychophsyics, 1969, 6, 105-110.

\section{NOTE}

1. McCollough, C., \& Clark, D Colorimetric data on border-contingent color aftereffects: II. Dependency of aftereffect hue on inspection colors. 1970 , unpublished work.

2. An alternative explanation to these results was suggested by Stromeyer (1969), who postulated an opponent color linkage between orthogonal orientation detectors.

(Received for publication August 13, 1971.) 